\title{
Congenital malignant neurilemmoma
}

\author{
The late MARTIN BODIAN AND ANDREW W. WILKINSON \\ From The Hospital for Sick Children, \\ Great Ormond Street, London
}

SYNOPSIS A unique case of a congenital malignant neurilemmoma in a newborn baby is describef

Congenital neoplasms are rare; Wells (1940) found only four instances (all neuroblastoma) among 3,000 necropsies on newborn infants. Review of the published reports has revealed only 66 cases in which the diagnosis was established beyond possible doubt; among these there were 23 cases of neuroblastoma and 33 of congenital sarcoma. Most of the latter were recorded as round-cell or spindle-cell sarcoma but none was recognized as congenital neurilemmoma. During the period 1925 to 1961 inclusive 1,106 cases of malignant neoplasm (excluding leukaemia) were seen at The Hospital for Sick Children, Great Ormond Street, and of these 24 were congenital neoplasms; among these there were five neuroblastomas, 13 teratomas, and three sarcomas, including the case reported in this paper. This is the only instance of malignant neurilemmoma seen at this hospital. Stout (1935) reported 50 patients with neurilemmomas, the youngest of whom was aged 1 year and none of the tumours showed any evidence of malignancy.

\section{CASE HISTORY}

At the birth of a girl born on 12 March 1959 a tumour was observed on the medial aspect of the right forearm extending from the front of the elbow two-thirds of the way to the wrist. The tumour was soft in consistency, bluish red in appearance, not involving the skin but attached on the deep aspect to the muscles of the forearm; it was not tender, there was no pulsation, and glands were not felt in the cubital fossa or axillae. The provisional diagnosis was haemangioma or lymphangioma. At operation at the age of 9 days (C. F. Swinton), an incision along the medial side of the forearm was carried across the front of the elbow. The tumour mass was found deep to the deep fascia; it appeared to be encapsulated and was bluish opalescent in colour. Bleeding was considerable and appeared to be infiltrating the muscles of the common flexor origin. The tumour could be shelled out from its surroundings except at its deep surface where it was attached to the muscles. When the capsule was opened the material of the tumour was gelatinous in consistency. The histological appearances suggested that this was a sarcoma and the baby was referred to The Hospital for Sick Children for advice.

On further examination no abnormality was found. apart from the incision in the right forearm witk surrounding inflammation and induration. The live spleen, and kidneys were not palpable. No abnormalities were seen in a radiograph of the chest and an intra muscular pyelogram showed good concentration of tho medium by both kidneys and no evidence of any renat abnormality. Abnormal calcification was not seen ip radiographs of the abdomen. Re-examination of the tumour excised from the forearm suggested that the appearances might be those of a metastatic neuroy blastoma of primitive cellularity and high mitotio activity with evidence of necrosis in some areas. Treat ment with vitamin $B_{12}$ was begun, $1 \mathrm{mg}$. being injecte $\bar{\Phi}$ intramuscularly on alternate days. It was also though desirable to explore the abdomen to make quite certain whether or not a primary tumour was present. $A \overline{Q Q}$ laparotomy the suprarenals were enlarged; there was no obvious sign of a tumour, the lymph glands at the base ợ the mesentery were enlarged, some were of pink and inflamed appearance. others were hard and white.
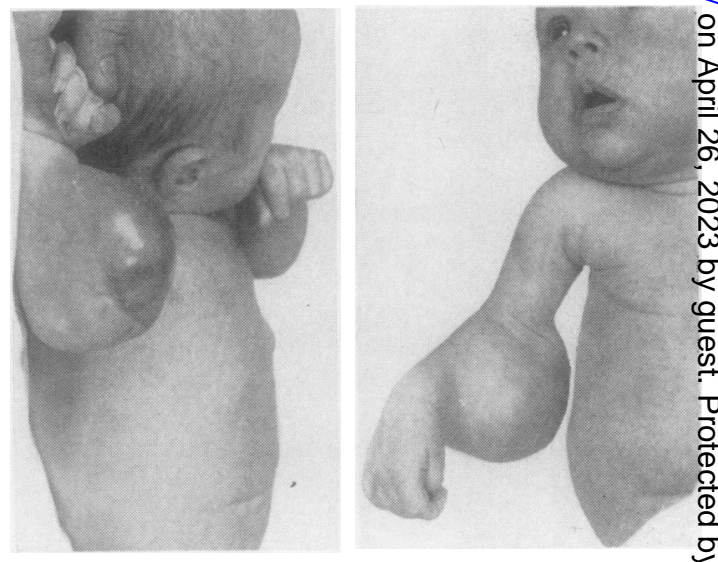

FIGS. 1 and 2. Size of first recurrent tumour on admissiộ before second operation. 


\section{Congenital malignant neurilemmoma}

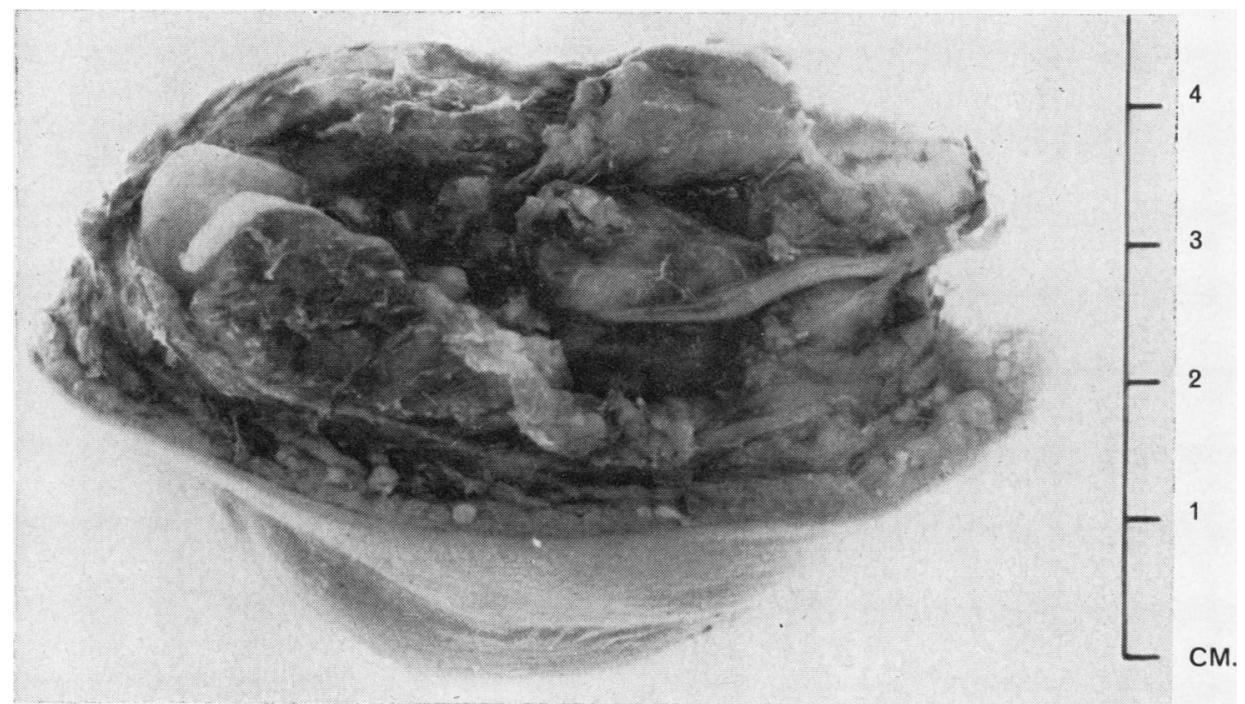

FIG. 3. Tumour excised at second operation showing the ulnar nerve running into the mass.

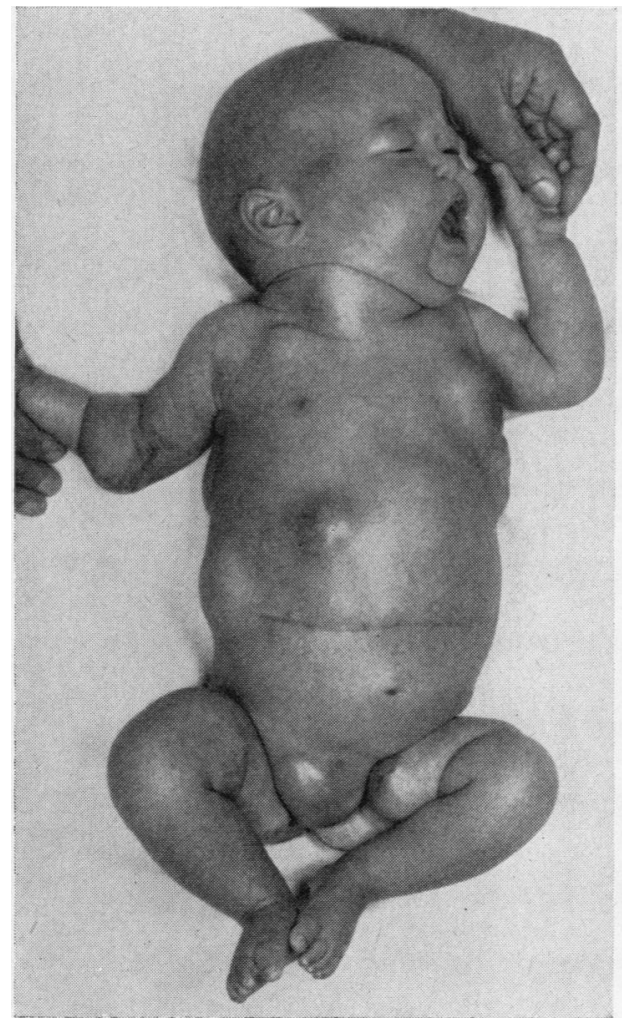

FIG. 4

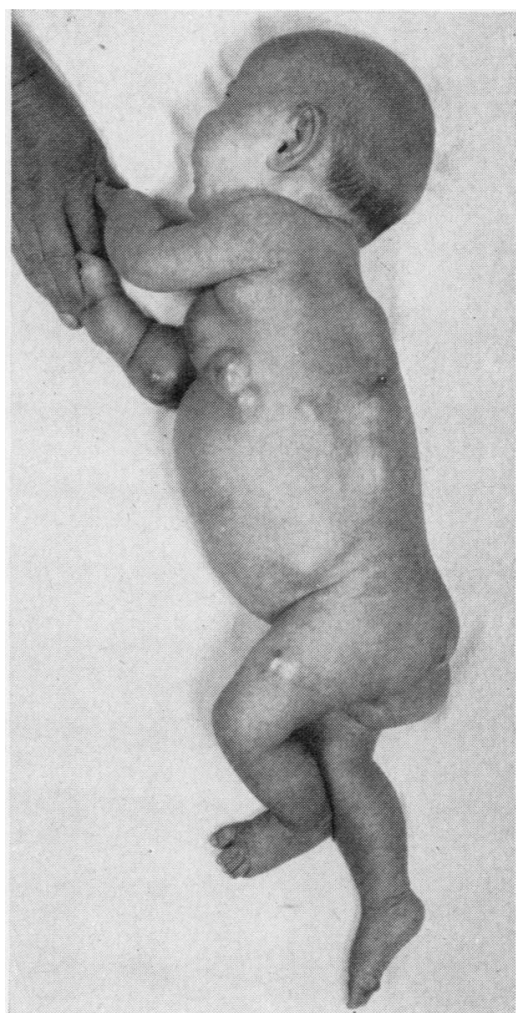

FIG. 5

FIGS. 4 and 5. Multiple metastases at the age of $3 \frac{1}{2}$ months. 
FIG. 6. Abdominal tumour masses, left hydronephrosis, left flat adrenal and right adrenal with tumour nodules.
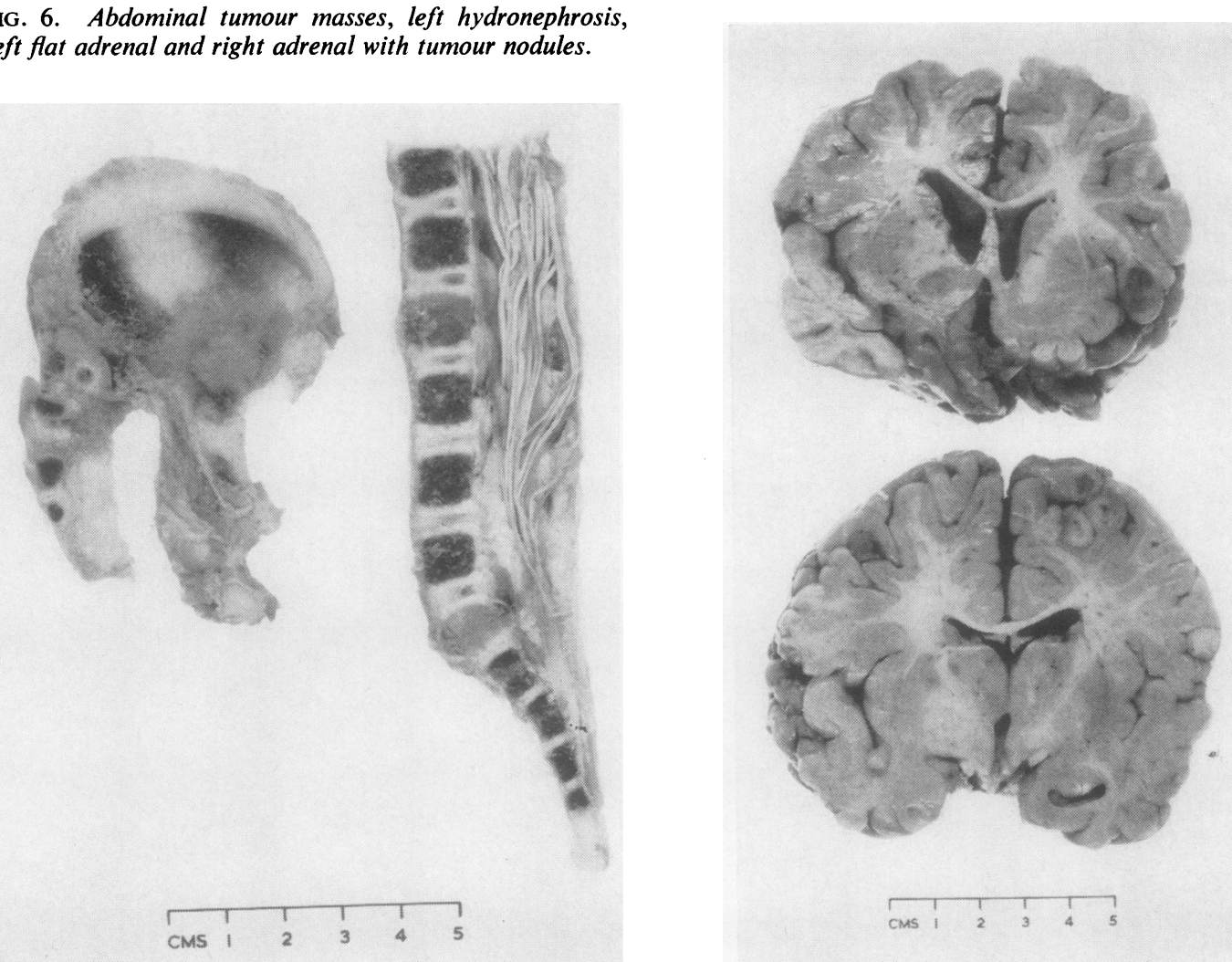

FIG. 7. Vertebral column and iliac bone with metastases.

FIG. 8. Brain with multiple metastases. 
gland was removed for histological examination. There being no other abnormality in the abdomen the wound was closed. Histological examination of the lymph node showed no evidence of neoplastic change but there were many eosinophil leucocytes in the medulla of the gland. A fortnight later the baby was discharged home, the vitamin $\mathbf{B}_{12}$ treatment being continued.

During the next 16 days the tumour of the forearm steadily increased in size until it occupied the upper five-sixths of the flexor aspect of the right forearm. It was highly vascular and appeared to be partly fluctuant. Glands were not felt in the axilla and the liver and spleen were not palpable. The baby was readmitted for local excision of the tumour. An elliptical incision encircled the mass in the forearm leaving a margin of $\frac{1}{2}$ in. of healthy skin on all sides and including the scar of the previous operation in the area to be removed. The deep fascia was opened along the volar surface of the forearm and many vessels were found beneath the deep fascia running into the tumour. The tumour infiltrated the flexor muscles and the dissection was carried through healthy muscle. The tumour extended backwards almost to the ulna and the ulnar nerve was identified, entering the upper deep aspect of the tumour from behind the medial epicondyle and leaving the lower end of the tumour. The nerve could not be dissected free of the tumour. The lower pole of the tumour at its deep aspect was freed by dividing the flexor muscles. The common flexor origin was divided above and the ulnar nerve was resected above and below the tumour, and the ulnar artery was ligated and divided. The excision of the tumour appeared to be complete. The baby was discharged a fortnight after operation and vitamin $B_{12}$ treatment was maintained. At the time of discharge one gland could be felt in the right axilla. This disappeared during the next fortnight but within six weeks of operation the baby was found to have swellings on the back, in the right groin, and in the left axilla. She was readmitted. There appeared also to be a recurrence of tumour at the upper end of the scar in the forearm. One swelling involved the right pubic tubercle and was hard and immobile. Another lay behind the areola of the left breast and the third was about $1 \frac{1}{2}$ in. in diameter and situated in the muscles of the right lumbar region just above the iliac crest. The lump in the back was excised and histological examination was thought to show a well-localized metastasis from a neuroblastoma. Swellings in the left breast and right groin continued to increase in size and a further swelling appeared below and lateral to the left breast. Dr. I. G. Williams was consulted and a course of radiotherapy was arranged at St. Bartholomew's Hospital. During the next three weeks a total dose of $1,100 \mathrm{r}$ was given to the region of the left breast, the right forearm, and the right pubis, and 600r to a further swelling which developed in the right hypochondrium. Other swellings appeared, two in the left groin and two over the lower ribs below the left scapula, one at the outer end of the laparotomy scar in the right groin, two in the right thigh and two in the left thigh, a large swelling just below the right buttock, and six over the lower thorax and lumbar region. All these metastases lay originally in the subcutaneous tissue, some were fixed to skin, others remained free. During the next fortnight in spite of the continuance of vitamin $B_{12}$ therapy and radiotherapy further metastases appeared on the trunk and limbs. The liver became palpably enlarged but the lung fields remained clear of metastases. Cortisone was given by injection, $100 \mathrm{mg}$. followed by $50 \mathrm{mg}$. b.i.d. This did not have any effect on the tumour. $X$-ray examination showed involvement by metastases of the mediastinum and of the right ileum. Radiotherapy was abandoned and the baby died at the age of 5 months.

\section{POST-MORTEM EXAMINATION}

EXTERNAL EXAMINATION There were many large tumour masses in both sides of the neck, extending into both supraclavicular regions and into the left axilla, to both sides of the chest, along both sides of the spine, overlying the left scapula, on the right upper abdominal wall, several close to the supraumbilical scar, the left hypochondrium, and both groins. There was a large mass involving most of the right thigh and a somewhat smaller mass in the left thigh. The right upper extremity was considerably distorted and there was a very large mass in the region of the right elbow posteriorly, with a scar extending below it to the wrist. This tumour mass was slightly ulcerated and reddish-blue in appearance.

INTERNAL EXAMINATION There were many neoplastic deposits in the calvarium and base of the skull, and where the tumour had invaded the bone it showed a dense sclerotic pale cream appearance. Infiltration was particularly marked in the sphenoid bone, and extended into the sella turcica and into the pituitary gland. The brain was slightly heavier than normal and many nodules of up to $1 \mathrm{~cm}$. diameter were seen in both sides of the cerebral cortex, in the basal ganglia, and in the right cerebral hemisphere. There was no dilatation of the ventricles.

The third, fifth, and eleventh dorsal and second lumbar vertebrae showed sclerosing neoplastic involvement of the vertebral bodies.

Large multilobed and partly necrotic masses were found in both supraclavicular regions involving the cervical lymph nodes. These masses were larger on the right than the left.

There were many extrapleural masses in both sides of the thorax near the spine and close to the ribs.

The thymus, heart, and lungs were free from metastases.

The diaphragm showed multiple large tumour nodules on both pleural and peritoneal aspects.

The liver appeared normal, and so did the gall bladder and bile ducts.

The pancreas showed a moderately large tumour deposit in its tail.

The spleen was normal. 


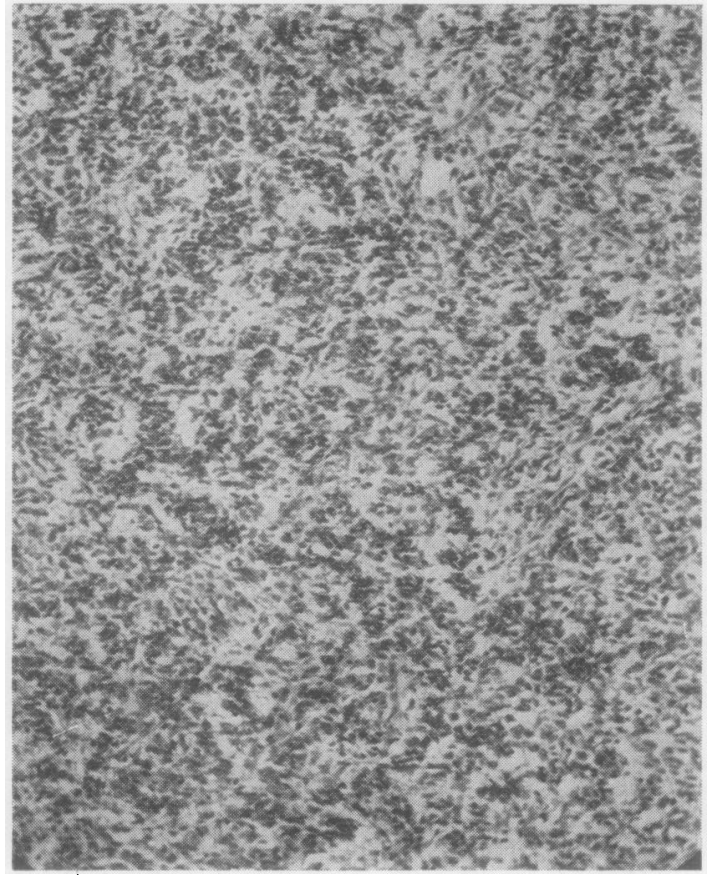

FIG. 9.

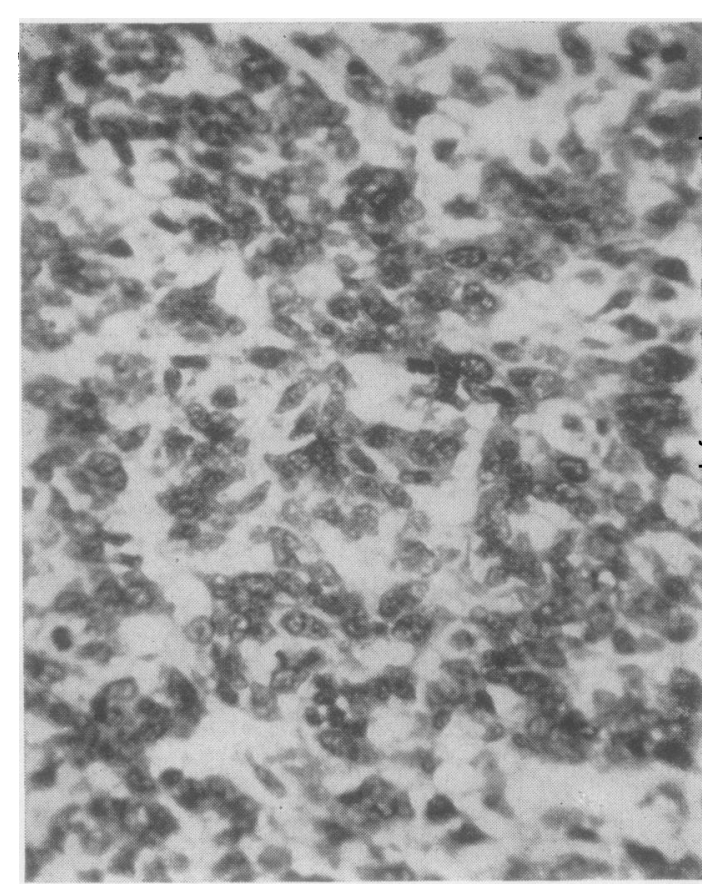

FIG. 10 .

FIG. 9. Photomicrograph of tumour $\times 90$ with suggestion of pallisading of cells.

FIG. 10. Photomicrograph of tumour $\times 360$.

The adrenal glands were not involved in the neoplastic process. Large tumour nodules were found both above and below the right adrenal. The left adrenal was very flat and lay in close association with a large tumour mass at the upper pole of the left kidney, which was concave and flattened.

The right kidney was not enlarged and contained three small whitish tumour nodules. The left kidney was much enlarged and surrounded by a huge tumour mass which measured $14 \times 11.5 \mathrm{~cm}$., was largely necrotic, and was retroperitoneal in position.

The ureters were normal. Several small tumour nodules were found in the adventitia of the urinary bladder. The genito-urinary tract was normal.

Many of the tumour masses mentioned in the description of the external examination were related to subcutaneous tissues and muscles but not to bony structures.

In addition to the multiple tumour deposits in the skull and spine the pelvic bones showed multiple densely sclerosed secondary deposits, particularly the iliac bones, but the sternum, ribs, right radius, ulna, and humerus showed no neoplastic involvement.
HISTOLOGY The sections of the primary tumouro recurrence, and metastases showed similar features, but the post-mortem material displayed much higher mitotic activity than the surgical specimens.

The tumour was densely cellular, with typicas regimentation of the cells which were spindle shaped or ovoid with a moderate amount of fibrillary. material staining for reticulin and collagen.

DISCUSSION

What appears to be a unique example of a cop genital malignant neurilemmoma has been described: A striking clinical feature of this tumour was its apparently close relationship to the ulnar nerve. A neuroblastoma arising in a peripheral nerve has never been described and seems to be even less likely than a congenital malignant neurilemmoma. The persistent and widespread recurrence of this tumout was a good indication of its highly malignant nature

\section{REFERENCES}

Stout, A. P. (1935). Amer. J. Cancer, 24. 751.

Wells, H. G. (1940). Arch Path., 30, 535. 